\title{
Injection of Inner Oort Cloud Objects into the Distant Kuiper Belt by Planet Nine
}

\author{
Konstantin Batygin (iD and Michael E. Brown (iD) \\ Division of Geological and Planetary Sciences California Institute of Technology, Pasadena, CA 91125, USA \\ Received 2020 December 30; revised 2021 February 24; accepted 2021 March 11; published 2021 April 6
}

\begin{abstract}
The outer solar system exhibits an anomalous pattern of orbital clustering, characterized by an approximate alignment of the apsidal lines and angular momentum vectors of distant, long-term stable Kuiper Belt objects. One explanation for this dynamical confinement is the existence of a yet-undetected planetary-mass object, "Planet Nine (P9)." Previous work has shown that trans-Neptunian objects, that originate within the scattered disk population of the Kuiper Belt, can be corralled into orbital alignment by Planet Nine's gravity over $\sim$ Gyr timescales, and characteristic P9 parameters have been derived by matching the properties of a synthetic Kuiper Belt generated within numerical simulations to the available observational data. In this work, we show that an additional dynamical process is in play within the framework of the Planet Nine hypothesis, and demonstrate that P9-induced dynamical evolution facilitates orbital variations within the otherwise dynamically frozen inner Oort cloud. As a result of this evolution, inner Oort cloud bodies can acquire orbits characteristic of the distant scattered disk, implying that if Planet Nine exists, the observed census of long-period trans-Neptunian objects is comprised of a mixture of Oort cloud and Kuiper Belt objects. Our simulations further show that although inward-injected inner Oort cloud objects exhibit P9-driven orbital confinement, the degree of clustering is weaker than that of objects originating within the Kuiper Belt. Cumulatively, our results suggest that a more eccentric Planet Nine is likely necessary to explain the data than previously thought.
\end{abstract}

Unified Astronomy Thesaurus concepts: Trans-Neptunian objects (1705); Planetary dynamics (2173); Oort cloud objects (1158)

\section{Introduction}

Over the course of the past three decades, the dynamical architecture of the solar system's trans-Neptunian region has steadily come into sharper focus. In turn, the unprecedented level of detail unveiled by observational surveys has repeatedly challenged our understanding of the solar system's markup, as well as the hitherto standard model of its long-term evolution (Saillenfest 2020). The resulting paradigm shift toward an instability-driven model of the solar system's early dynamics has resolved much of the tension between theory and observations, and today, the physical mechanisms that shaped the structure of the proximate $(a \lesssim 50 \mathrm{au})$ Kuiper Belt have been broadly delineated (Morbidelli \& Nesvorný 2020 and the references therein). In contrast, a full understanding of the anomalous orbital architecture exhibited by distant transNeptunian objects-and its dynamical origins-remains elusive.

Perhaps the most striking peculiarity displayed by the extended Kuiper Belt is the approximate alignment of longperiod $\left(P \gtrsim 4000 \mathrm{yr}\right.$ ) orbits in physical space ${ }^{1}$ (Batygin \& Brown 2016). That is, trans-Neptunian objects with semimajor axes in excess of 250 au and inclinations smaller than $40 \mathrm{deg}$ have apsidal lines and angular momentum vectors that cluster together in an unexpected manner (Figure 1). Although this clustering is visible by eye, it is important to note the degree of orbital confinement is not uniform, and instead depends sensitively on dynamical stability. In particular, objects that experience comparatively rapid orbital diffusion due to periodic

\footnotetext{
1 Simultaneous alignment of orbital planes and eccentricity vectors requires the orbits to have similar longitudes of perihelion, $\varpi$, inclinations, $i$, and longitudes of ascending node, $\Omega$. Importantly, we note that the grouping of the arguments of perihelion, $\omega=\varpi-\Omega$, was first pointed out by Trujillo \& Sheppard (2014).
}

interactions with Neptune (shown as green ellipses on Figure 1) are much less tightly confined than their stable and metastable counterparts $^{2}$ (shown as purple and gray ellipses in Figure 1, respectively).

A number of explanations have been proposed for the origins of the alignment depicted in Figure 1. Arguably the most rudimentary of them is the notion that the alignment is not real, and is instead a figment of observational bias or statistical chance. To this end, individual surveys - which have only searched limited areas of the sky-have been unable to overcome their own observational biases sufficiently to rigorously determine the absence or presence of orbital alignment (Shankman et al. 2017; Bernardinelli et al. 2020; Napier et al. 2021). Nevertheless, a combined observability analysis of all available data shows that, after taking observational biases into account, distant KBOs are jointly clustered in eccentricity and angular momentum vectors at the 99.8\% significance level (Brown 2017; Brown \& Batygin 2019). Thus, if we interpret the picture insinuated by the data at face value, the observed clustering requires a sustained gravitational torque that maintains orbital alignment against differential precession induced by Jupiter, Saturn, Uranus, and Neptune.

In principle, such a torque could come from any distant massive object, including a lopsided massive disk of icy material (Madigan et al. 2018; Sefilian \& Touma 2019), a primordial black hole (Scholtz \& Unwin 2020), an asymmetric dark matter halo, etc. In this work, we opt for what we consider

\footnotetext{
2 For the purposes of this work, we define the tiers of orbital stability as follows: if more than $20 \%$ of the clones of a given object eject over the course of a 4 Gyr integration, an object is deemed unstable. If more than $20 \%$ of the clones of a given object change their semimajor axes by a factor of two during a 4 Gyr integration but do not eject, an object is deemed metastable. If neither of these events occurs, we deem such an object stable.
} 


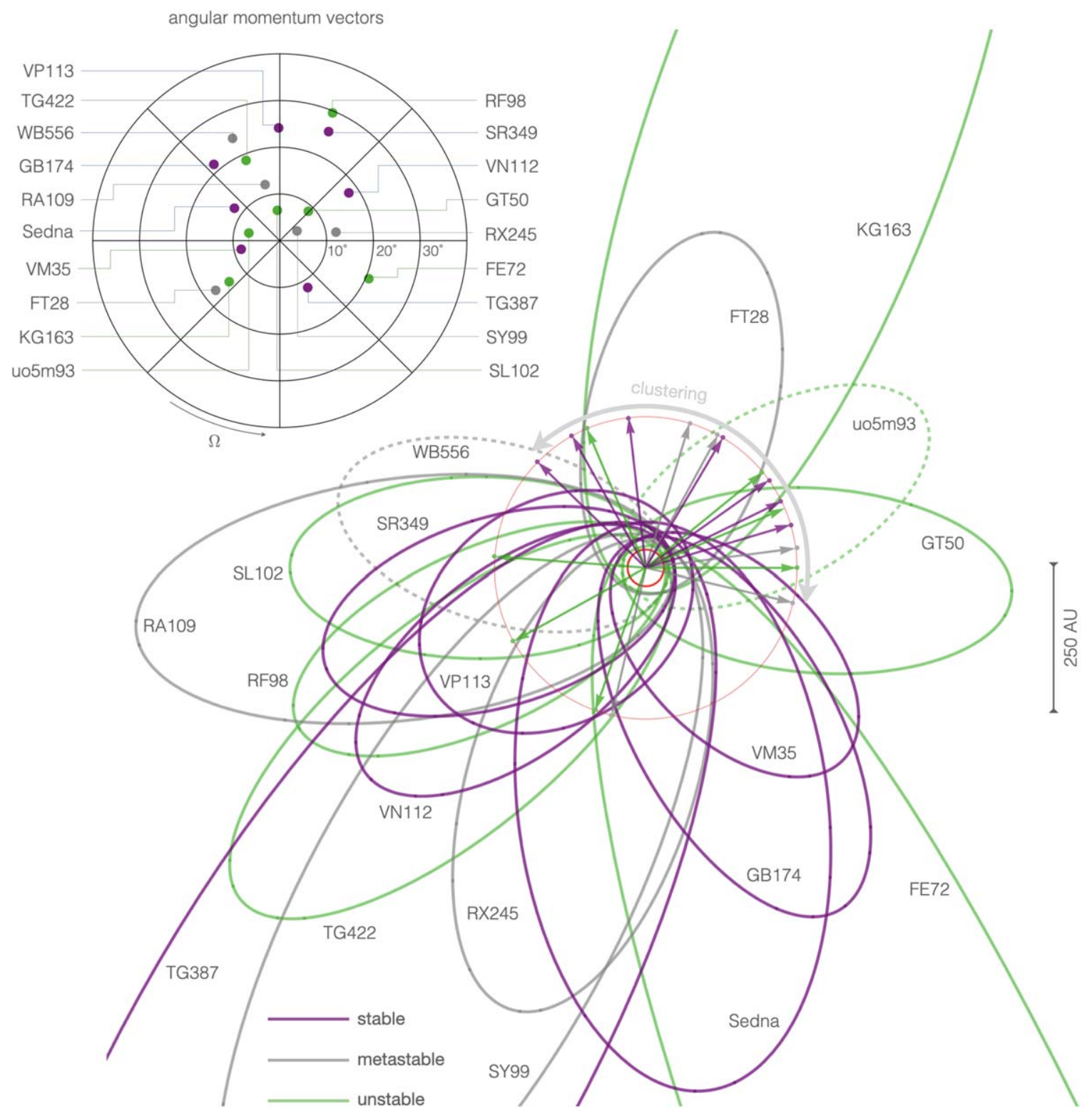

Figure 1. Census of trans-Neptunian objects with $a>250 \mathrm{au}, q>30 \mathrm{au}$, and $i<40 \mathrm{deg}$. The orbits of objects that experience rapid orbital diffusion are shown in green, while the orbits of stable and slowly diffusing KBOs are depicted in purple and gray, respectively. The dotted orbits correspond to KBOs whose parameters and uncertainties are not reported in the JPL Small Body Database, and whose stability was estimated from a single 4 Gyr integration of the nominal orbit rather than similar integrations of 10 clones of each object. The $i-\Omega$ polar inset informs the tilt of the orbital angular momentum vectors. Stable and metastable objects have apsidal lines that cluster around $\langle\varpi\rangle \sim 60 \mathrm{deg}$, and form a mean plane that is inclined with respect to the ecliptic by $\langle i\rangle \sim 10 \mathrm{deg}$, with a mean longitude of ascending node of $\langle\Omega\rangle \sim 90 \mathrm{deg}$.

a somewhat less exotic alternative, and envision that the alignment of distant orbits is sustained by a yet-unseen planet, "Planet Nine," occupying a mildly eccentric, slightly inclined orbit with a period of order $\sim 10,000 \mathrm{yr}$ (Batygin \& Brown 2016; Brown \& Batygin 2016). We note, however, that because the dominant mode of P9-induced dynamics is secular (i.e., stemming from phase-averaged gravitational potential of P9), our results are not sensitive to the composition of P9—only its mass and its orbit.

\section{Hypothesis}

In a recent effort (Batygin et al. 2019), we have carried out an extensive suite of $N$-body simulations of the solar system's long-term evolution in the presence of Planet Nine, and have demonstrated that a synthetic Kuiper Belt sculpted by a $m_{9}=5 M_{\oplus} \mathrm{P} 9$ residing on a $a_{9}=500 \mathrm{au}, e_{9}=0.25$, and $i_{9}=20 \mathrm{deg}$ orbit adequately matches the observations. A subtle limitation of these calculations, however, is that they treat the solar system as an isolated object, ignoring the effects of passing stars, both during the solar system's infancy and during its long-term field evolution, as well as the gravitational tides of the birth cluster and the Galaxy. As such, all of the material that comprises the distant $(a \gtrsim 250$ au) Kuiper Belt within the framework of these numerical experiments is envisioned to have been sourced from inside of $30 \mathrm{au}$, and emplaced into the trans-Neptunian region by scattering off of the giant planets during the solar system's early transient instability (Levison et al. 2008; Nesvorný 2018).

Although the aforementioned picture reasonably represents the evolution of objects with semimajor axes on the order of a 
few hundred astronomical units, more recent detections of trans-Neptunian objects (Sheppard \& Trujillo 2016; Sheppard et al. 2019) increasingly point to a pronounced abundance of long-period TNOs with $a \gtrsim 1000$ au. Crucially, this orbital domain borders the inner Oort cloud (IOC) - a population of debris captured onto detached, long-term stable orbits during the solar system's residence within its birth cluster (Kaib et al. 2011; Clement \& Kaib 2020). The marked existence of such extremely long-period members of the Kuiper Belt demands that we consider the possibility that a fraction of the objects that comprise the population shown in Figure 1 have been injected into the distant solar system from the outside, through an intricate interplay between Galactic effects and Planet Nine's gravity.

In this work, we investigate this hypothesis through numerical experimentation, with an eye toward understanding how the process of mixing between the outer Kuiper Belt and the IOC alters the degree of orbital confinement facilitated by Planet Nine. In particular, we simulate the formation of the inner Oort cloud within the solar system's birth cluster (Section 3) and subject the resulting population of debris to P9-forced evolution (Section 4). As we describe below, our results indicate that although material injected into the distant Kuiper Belt by Planet Nine exhibits orbital clustering, the degree of confinement is inferior compared to that exhibited by objects that originate within the scattered disk. These findings introduce an added degree of uncertainty into our attempts to accurately estimate the physical and orbital properties of Planet Nine.

\section{Formation of the Inner Oort Cloud}

Statistically speaking, the vast majority of stars form in stellar associations (Lada \& Lada 2003; Porras et al. 2003). The solar system is no exception to this rule, and the enrichment of meteorites in short-lived radiogenic isotopes is among the most direct lines of evidence that point to the notion that the Sun's birth environment likely played an important role in shaping the solar system's large-scale structure (see Adams 2010 for a review). In fact, if the process of planetary accretion unfolded while the Sun was still embedded within its birth cluster, the formation of a perihelion-detached cloud of debris would have been all but inevitable.

An inescapable consequence of the solar system's residence within a cluster of stars is that at sufficiently large orbital radii, passing stars and the cluster's mean-field potential exert significant perturbations on solar system objects. Correspondingly, any planetesimals that would have been scattered out to large heliocentric distances by the forming giant planets would have experienced some degree of modulation by cluster effects (Morbidelli \& Levison 2004). In particular, published numerical simulations show that as long as the solar system continues to reside within its birth cluster, the population of planetesimals that attain $r \gtrsim 1000$ au through outward scattering remain entrained in a quasi-steady state cycle of enrichment through giant planet scattering, circularization through cluster effects, and stripping by passing stars (Brasser et al. 2006, 2012). As soon as the Sun leaves its birth environment, however, this process ceases to operate, terminating the generation of the IOC.

We have simulated this sequence of events depicted in Figure 2, following the procedure outlined in Brasser et al. (2006). Our simulations included Jupiter and Saturn, residing at
$a_{\mathrm{J}}=5.5 \mathrm{au}$ and $a_{\mathrm{S}}=9 \mathrm{au}$, respectively, as well as a sea of $N=10^{5}$ massless planetesimals, spanning the 4.5-12 au range in heliocentric distance. Both of the planets as well as the test particles were initialized on circular and coplanar orbits, and were integrated using the conservative variant of the BulischStoer algorithm implemented in the mercury 6 gravitational dynamics software package (Chambers 1999). The initial time step and integration accuracy parameter were set to $\Delta t=10^{2}$ days and $\epsilon=10^{-12}$ respectively.

In addition to planetary perturbations, we accounted for the effects of passing stars with impact parameters smaller than $b_{\star} \leqslant 0.1 \mathrm{pc}$, as well as the mean-field potential of the solar birth cluster. For definitiveness, we modeled the cluster as a Plummer sphere, setting the total mass to $M_{\infty}=1200 M_{\odot}$ and Plummer radius to $c=0.35 \mathrm{pc}$ in an effort to approximately mimic the properties of the Orion Nebular Cluster ${ }^{3}$ (see also Batygin et al. 2020 and the references therein). We further assumed the Sun to steadily orbit the cluster core at the halfmass-radius of $r_{\odot}=\left(1+2^{1 / 3}\right) / \sqrt{3} c \approx 0.33 \mathrm{pc}$ on a circular orbit, and ignored the long-term dissipation of the system, thereby holding the stellar number density fixed at $n_{\odot} \approx 1400 \mathrm{pc}^{-3}$. Finally, we adopted a velocity dispersion of $\langle v\rangle=1 \mathrm{~km} \mathrm{~s}^{-1}$ for all 19 stellar species ${ }^{4}$ that comprised the model stellar mass function of Heisler et al. (1987), and intermittently injected passing stars into the simulation domain following the method outlined in Heisler et al. (1987). Unlike these authors, however, we did not employ the impulse approximation, and instead resolved stellar flybys through selfconsistent $\mathrm{N}$-body integration (adopting the simulation parameters described above), setting the stellar injection/ejection radius to $0.1 \mathrm{pc}$. Cumulatively, these choices yield a characteristic stellar encounter rate of $\Gamma \sim n_{\odot} \pi b_{\star}^{2}\langle v\rangle \sim 50 \mathrm{Myr}^{-1}$.

For completeness, we note a number of additional simplifying assumptions that were made in our calculations. First, we assumed an essentially perfect star formation efficiency, such that the total mass of the cluster is equal to the mass comprised by the stars. In practice, this correspondence is of little dynamical consequence, since Kozai-Lidov type variations in particle parameters due to the cluster's mean-field potential (see, e.g., Section 2 of Batygin et al. 2020) represent only a small, longer-term correction to direct perturbations exerted by the stars, meaning that modulating the mass budget of the gas is unlikely to appreciably change the simulations' outcomes. Second, we approximated the IMF within the cluster as being similar to the present-day mass function with the exception of omitting white dwarfs. While a more thorough treatment of the IMF is possible, this assumption is unlikely to manifest as the dominant source of error in our calculations. Finally, we ignored the potential presence of P9 itself-and its accompanying perturbations-during the cluster stage. This simplification can be justified through a timescale argument: published simulations indicate that the P9-induced evolution unfolds on $\sim$ Gyr timescales - far longer than lifetimes of a typical birth cluster. This means that even if Planet Nine were extant during the Sun's cluster phase, its gravity would not

\footnotetext{
3 Note that these choices correspond to a cluster core radius of $r_{\mathrm{c}} \approx 0.23 \mathrm{pc}$ and central number density of $n_{\mathrm{c}} \approx 1.7 \times 10^{4} \mathrm{pc}^{-3}$-values comparable to the properties of the Trapezium cluster, embedded within the ONC. Moreover, with these parameters, the mean stellar number density interior to the $M / M_{\infty}=95 \%$ radius evaluates to $\langle n\rangle \approx 100 \mathrm{pc}^{-3}$, in agreement with observations (Hillenbrand \& Hartmann 1998).

4 White dwarfs were neglected from the stellar mass function.
} 


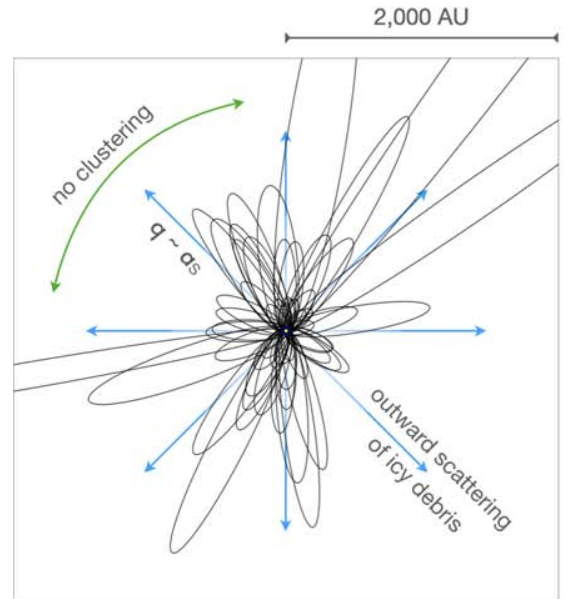

giant planet formation epoch

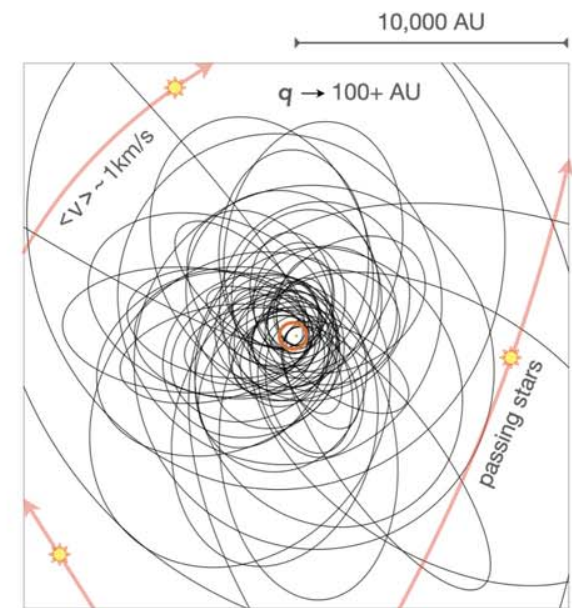

passing stars perturb debris at $1000+\mathrm{AU}$

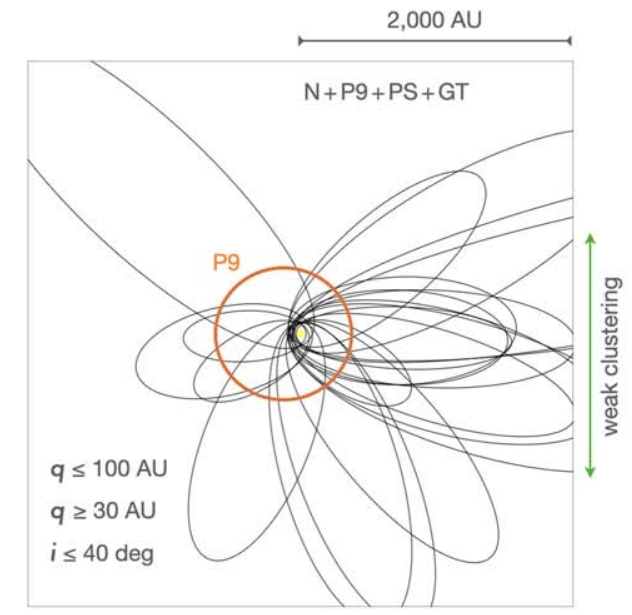

distant bodies injected back into the Kuiper belt

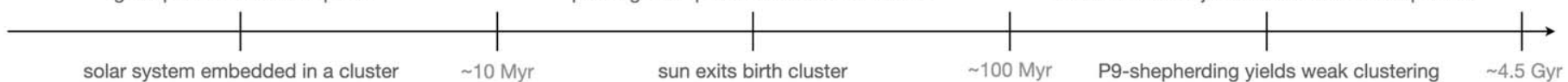

Figure 2. Sequence of events modeled within this work. A perihelion-detached population of trans-Neptunian objects forms while the Sun is embedded within its birth cluster. Subsequently, over the multi-Gyr lifetime of the solar system, Planet Nine slowly diminishes the perihelia of a subset of these extremely long-period objects, mixing them into the observed census of Kuiper Belt objects. Orbits shown on the left and middle panels of the figure are taken from the $t=4$ Myr model of the inner Oort cloud (IOC) shown in Figure 3. Orbits depicted on the right panel of the figure correspond to the same sample of orbits evolved for $t>2$ Gyr, and shown as dynamical footprints Figure 4.

constitute an efficient perihelion-detachment mechanism for outward-scattered planetesimals.

To smooth over statistical fluctuations inherent to each recapitulation of stochastic evolution facilitated by stellar encounters, we split the test particle population of our model into 10 separate disks (each composed of $N=10^{4}$ particles), and integrated each system within a distinct realization of the cluster. Each integration was carried out over a timespan of $\tau=10$ Myr. This timescale is simultaneously comparable to the characteristic lifetime of a typical protoplanetary disk (Haisch et al. 2001), and close to the upper bound on the Sun's permissible residence time within the model cluster. Importantly, the latter constraint comes from a dynamical limit on the product of stellar number density and cluster residence time of $\langle n\rangle \tau \lesssim 2 \times 10^{4} \quad \mathrm{Myr} \mathrm{pc}^{-3}$, derived from the inclination dispersion of the cold classical Kuiper Belt (Batygin et al. 2020), and is largely independent of the processes modeled here. With the adopted parameters, we found that a substantial IOC forms within $\sim 1 \mathrm{Myr}$, and reaches steady state in terms of particle count at $\sim 2$ Myr. More specifically, between 2 and $10 \mathrm{Myr}$, the number of particles with semimajor axes in excess of $40 \mathrm{au}$ and perihelion distance greater than $40 \mathrm{au}$ fluctuates between 200 and 300, although it is important to understand that the particles that comprise the cloud are continuously in flux.

A representative snapshot of the dynamical state of the IOC at $t=4 \mathrm{My}$ is shown in Figure 3. The top left panel depicts particle eccentricities as a function of semimajor axes. Constant-perihelion curves corresponding to $q=5,10,20$, 30 , and $40 \mathrm{au}$ are also outlined, and the outward transport of particles along the Saturnian scattered disk is evident. Objects with $q \leqslant 40$ au are shown as white circles, while perturbed particles with $q>40$ au are denoted with filled points, each color coded in accord with the cluster realization within which it was generated. A smoothed orange density histogram underlying the perihelion-detached population of particles is also illustrated in each panel. Particle inclinations are depicted in the top right panel of Figure 3 and show that although stellar encounters can generate retrograde objects, the kernel of the IOC is comprised of objects with inclinations on the order of a few tens of degrees.

The argument of perihelion and longitude of ascending node are portrayed in the bottom left and bottom right panels, respectively. While the nodal structure of the cloud is fully randomized, the argument of perihelion exhibits distinct clustering around $\omega=0$ and $\omega=180 \mathrm{deg}$. Unlike the dynamical grouping shown in Figure 1, these patterns are readily understood. Recall that within the context of our simulations, particles attain large heliocentric distances exclusively by scattering off of giant planets, meaning that by the time they get perturbed by passing stars, their orbits are nearly radial but planar, with $e \sim 1-q_{\mathrm{S}} / a \sim 0.99$ and $i \sim 0$. Because $\Omega$ is illdefined at $i=0$, even a weak stellar flyby can drastically alter the longitude of ascending node. Significantly changing $\omega$ on the other hand, would require a perturbation to rotate the nearly radial orbit away from the orbital plane of Jupiter and Saturn by a large angle. Instead, Kepler's second law insinuates that interactions are most likely to occur at aphelion, where an impulsive change in particle velocity can easily modulate the semimajor axis and eccentricity, but not alter the orbital orientation. As a result, even after being perturbed by passing stars, particles continue to come to aphelion close to the orbital plane of the giant planets, thereby maintaining $\omega=0$ or $\omega=180 \mathrm{deg}$.

The dynamical state of the IOC appears similar at other times in the integration, with the caveat that the inner and outer boundaries of the perturbed objects steadily march toward lower heliocentric distances in time, crudely tracking the everdecreasing impact parameter of the closest stellar encounter $b_{\min } \sim 1 / \sqrt{\pi n_{\odot}\langle v\rangle t}$. Overall, we find that the dynamical decoherence timescale of the detached population is somewhat shorter than a million years, and adopt snapshots of the distant solar system at $1,2,3, \ldots, 10 \mathrm{Myr}$ as pseudo-independent models of the IOC. 

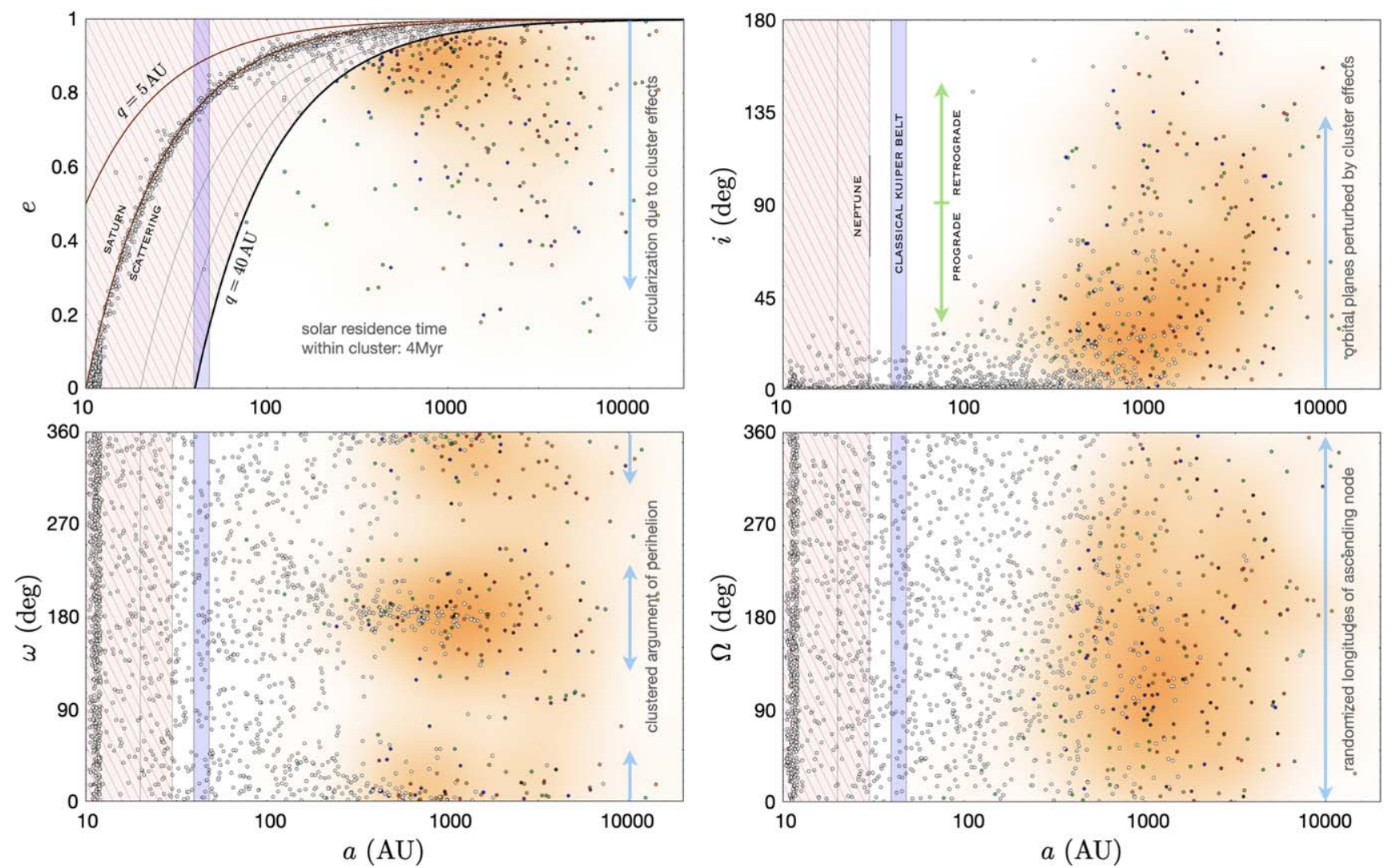

Figure 3. A representative inner Oort cloud created in our simulations. Multicolored points depict a perihelion-detached population of TNOs, generated within 10 distinct realizations of the model cluster. Crudely speaking, cluster effects facilitate perihelion lifting, excitation of inclination, and clustering of the argument of perihelion for $a \gtrsim 500$ au objects. As the Sun's cluster residence time increases, both the inner and outer edges of the detached population inch toward smaller heliocentric distances.

\section{P9-induced Evolution}

In absence of Planet Nine, the cloud of distant debris created by the interplay between giant planet scattering and cluster perturbations would essentially remain frozen over the mainsequence lifetime of the Sun. Gravitational perturbations due to P9, however, can steadily diminish the perihelia of such detached objects, bringing them into the fold of the scattered disk population of the Kuiper Belt. Here, we have simulated this process, self-consistently accounting for the $N$-body dynamics driven by Neptune, Planet Nine, and passing stars within the Galactic field (once again, following the methodology of Heisler et al. 1987), as well as the effect of the Galactic tide (implemented as in Nesvorný et al. 2017; see also Heisler \& Tremaine 1986; Wiegert \& Tremaine 1999; Clement \& Kaib 2020). As noted in the aforementioned references, this effect is most sensitive to the vertical component of the acceleration, which is largely determined by the mean local Milky Way disk density, which we set to $\rho_{\mathrm{MW}}=0.1 M_{\odot} \mathrm{pc}^{-3}$.

For definitiveness, we adopted the best-fit P9 parameters from Batygin et al. (2019): $m_{9}=5 M_{\oplus}, a_{9}=500 \mathrm{au}, e_{9}=0.25$, and $i_{9}=20 \mathrm{deg}$. Orbit-averaged effects of Jupiter, Saturn, and Uranus were also included in the calculations as an effective $J_{2}$ moment of the Sun. As discussed in Batygin et al. (2019), this numerical scheme constitutes an adequate compromise between computational cost and accuracy, allowing us to increase the initial time step in our simulations to $\Delta t=3000$ days.

We integrated each of the 10 variants of the IOC discussed in the previous section for $4.5 \mathrm{Gyr}$, and found that over the lifetime of the Sun, a significant fraction (that is, on the order of $20 \%)$ of the IOC gets injected into the distant $(a>250 \mathrm{au})$ Kuiper Belt, with approximately $10 \%$ injected in the final 2 Gyr. Dynamical footprints with $t>2 \mathrm{Gyr}$ of injected particles that satisfy crude stability and observability criteria (40 au $<\mathrm{q}<100 \mathrm{au}, i<40 \mathrm{deg}$ ) are shown in the top panels of Figure 4, where the color of the points informs the IOC model adopted for the initial conditions. More specifically, the panels on the left-hand side depict the particles' longitude of perihelion, relative to that of Planet Nine, while the polar plots on the right inform the angular momentum vectors of particle orbits.

Overall, IOC models that correspond to solar cluster residence time of $t \geqslant 2 \mathrm{Myr}$ yield consistent results, although the relevant particle count in each case is relatively small (Table 1). Thus, in order to more clearly portray the orbital distribution of the long-period Kuiper Belt generated by inward injection of distant debris, we show a smooth density histogram of our combined ${ }^{5}$ simulation data in the middle panels of Figure 4. The census of known long-period KBOs satisfying the same perihelion and inclination cuts is overplotted on the diagram, using the same color scheme as that in Figure 1. Finally, the bottom panels of Figure 4 depict the results of a control simulation using the same P9 parameters, but with particle initial conditions drawn uniformly from the $q \in(30$,

\footnotetext{
5 Although the distant Kuiper Belt sourced from the $t=1 \mathrm{Myr}$ model of the IOC shows virtually no apsidally anti-aligned objects with $q<100 \mathrm{au}$, the particle count is a factor of a few smaller than the other runs, meaning that its contribution is insignificant.
} 

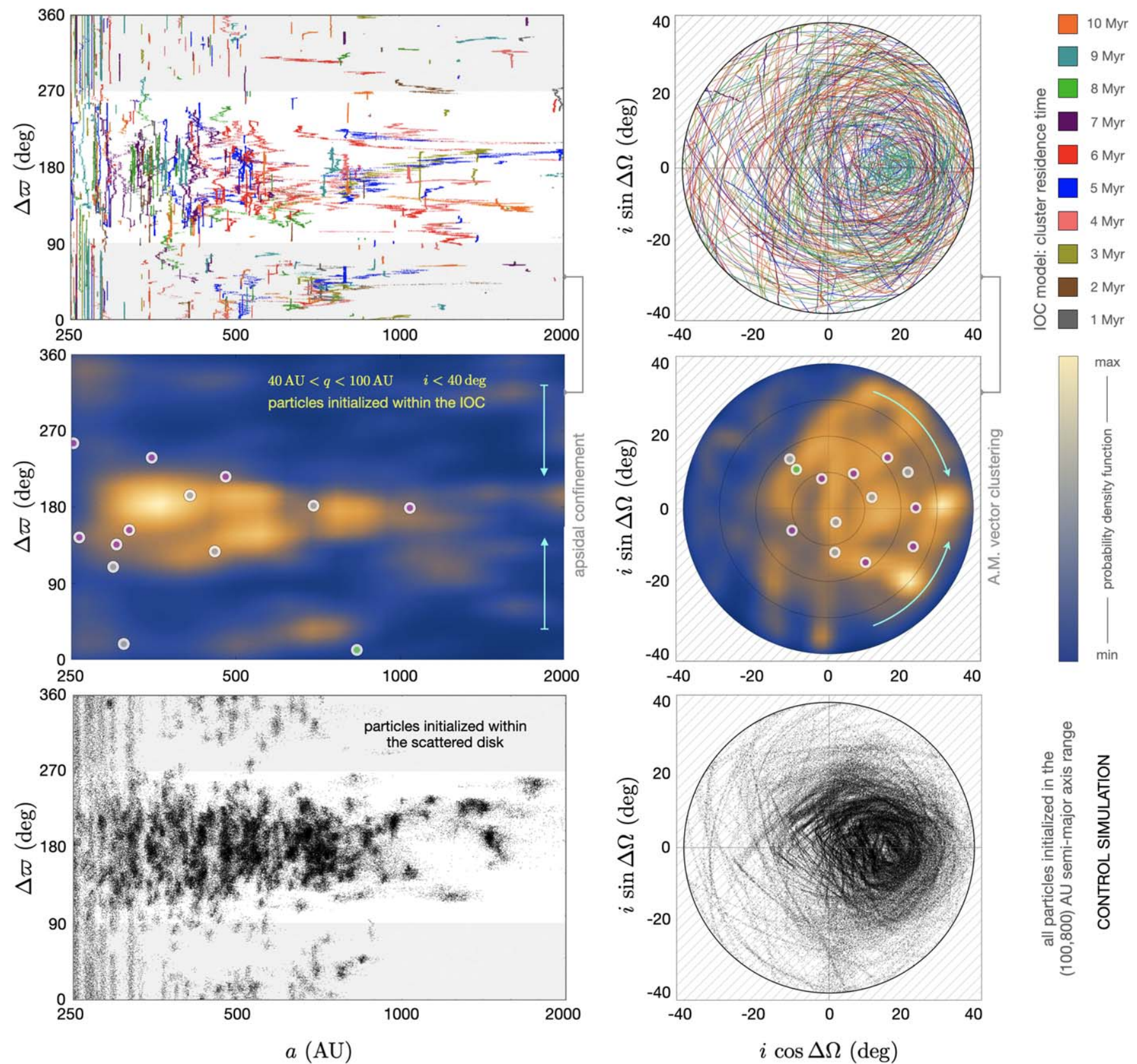

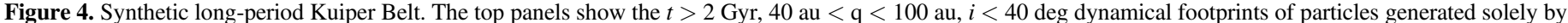

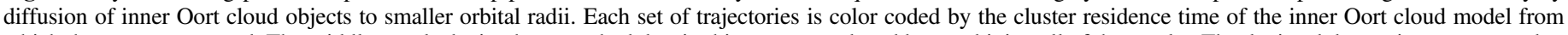

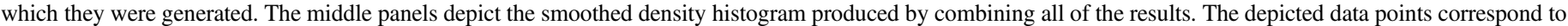

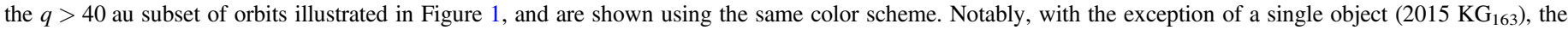

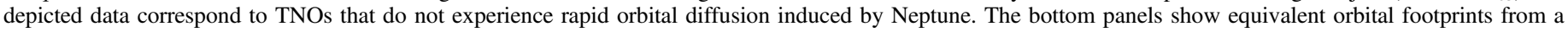

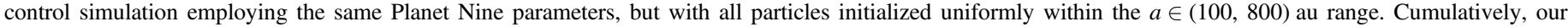

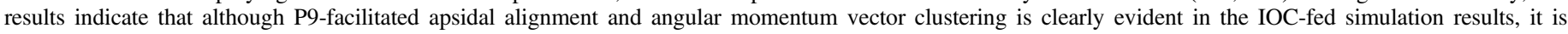
considerably weaker than orbital confinement exhibited by particles sourced from the scattered disk population of the Kuiper Belt.

100) au and $a \in ;(100,800)$ au ranges, and an initial inclination dispersion corresponding to a half-Gaussian distribution with a standard deviation of $15 \mathrm{deg}$.

Cumulatively, our simulations elicit two key results. First, the injected particles clearly follow typical P9-driven dynamical evolution (see Batygin \& Brown 2016; Batygin \& Morbidelli 2017; Hadden et al. 2018; Khain et al. 2018, 2020; Li et al. 2018) with relative longitude of perihelion exhibiting visible clustering around $\Delta \varpi \sim 180 \mathrm{deg}$ (Figure 4). Similarly, the inclination-node trajectories of these objects circulate around the P9-forced equilibrium in phase space, which can manifest in an apparent clustering of the longitudes of ascending node when viewed from the ecliptic. At the same time, it is important to note that the patterns of orbital clustering shown in Figure 4 are considerably less pronounced than those created in P9 simulations where distant KBOs are sourced entirely from the scattered disk.

To be more specific, the fraction of dynamical footprints that fall within \pm 90 deg of exact apsidal anti-alignment with respect to $\mathrm{P} 9$ in the middle panel of Figure 4 is a mere $f_{\varpi}=67 \%$. By comparison, our control simulation with initial semimajor axes of particles drawn from the $a \in(100 \mathrm{au}, 800 \mathrm{au})$ range yields 
Table 1

A Summary of Orbital Confinement Metrics for Each IOC-fed Simulation

\begin{tabular}{|c|c|c|c|c|c|}
\hline IOC Model & 1 & 2 & 3 & 4 & 5 \\
\hline$\overline{N^{\dagger}}$ & 11 & 15 & 15 & 26 & 37 \\
\hline$f_{\varpi}$ & $0.46(0 \%)$ & $0.62(0 \%)$ & $0.69(0 \%)$ & $0.64(0 \%)$ & $0.75(0 \%)$ \\
\hline$\langle i\rangle(\operatorname{deg})$ & $20.2(100 \%)$ & $9.2(40 \%)$ & $10.1(67 \%)$ & 4.6. (0\%) & $4.2(0 \%)$ \\
\hline IOC model & 6 & 7 & 8 & 9 & 10 \\
\hline$N^{\dagger}$ & 31 & 28 & 30 & 30 & 37 \\
\hline$\langle i\rangle / i_{\mathrm{rms}}$ & $0.29(0 \%)$ & $0.02(0 \%)$ & $0.14(0 \%)$ & $0.45(47 \%)$ & $0.13(0 \%)$ \\
\hline
\end{tabular}

Note. The percentages in parentheses indicate the fraction of down-sampled control simulations that yield metrics as low or lower than the reported numerical experiments.
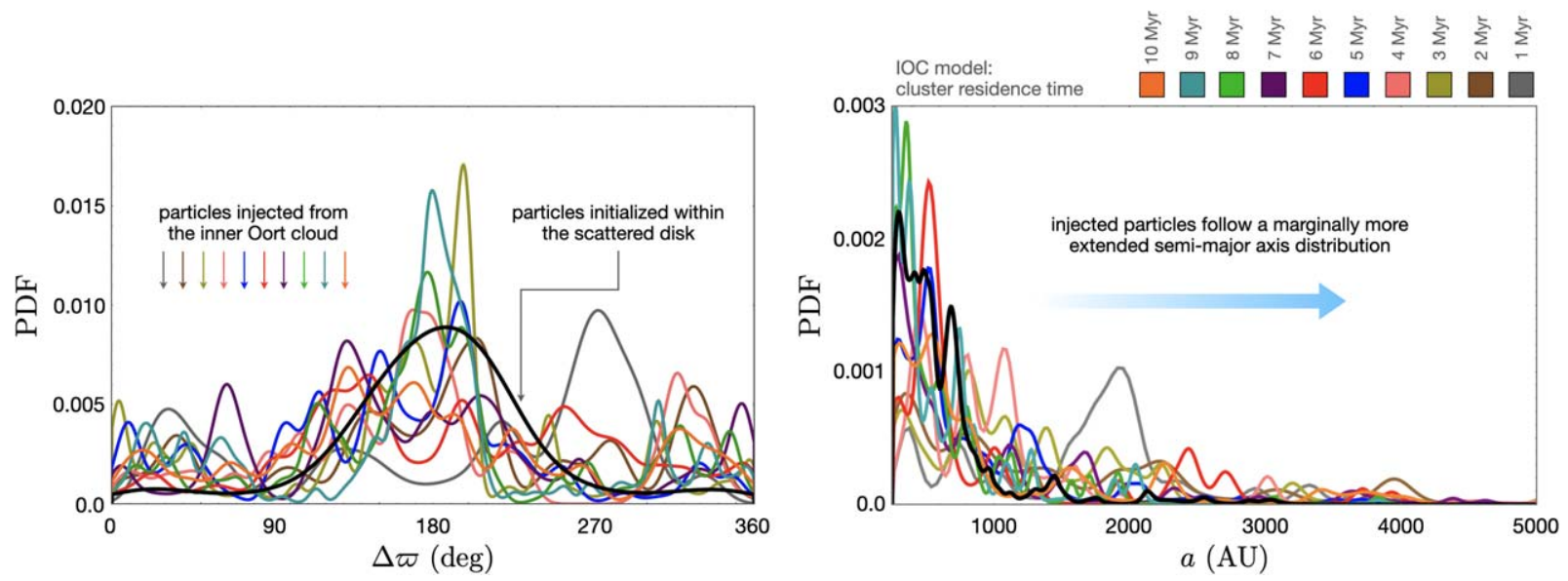

Figure 5. Apsidal clustering and semimajor axis distributions of simulated particles satisfying $a>250$ au, 40 au $<\mathrm{q}<100$ au, $i<40$ deg, and $t>2$ Gyr. The multicolored curves show the $\Delta \varpi$ and $a$ probability density functions of inward-injected Oort cloud objects obtained in our simulations. The black curves depict the probability density functions associated with synthetic KBOs that are initialized within the scattered disk, and evolved with identical $\left(m_{9}=5 M_{\oplus}, a_{9}=500\right.$ au, and $e_{9}=0.25, i_{9}=20 \mathrm{deg}$ ) P9 parameters. Although the dynamical forcing is the same, objects that originate within the Kuiper Belt exhibit significantly tighter apsidal clustering in our simulations than do injected IOC objects. Injected IOC objects further display a somewhat more extended semimajor axis distribution than our control simulation.

$f_{\varpi}=88 \%$. The confinement of angular momentum vectors in our IOC-fed simulations is similarly weak: the mean inclination and the associated rms dispersion exhibited by injected particles are $\langle i\rangle \approx 5 \mathrm{deg}$ and $i_{\mathrm{rms}} \approx 26 \mathrm{deg}$, respectively. Meanwhile, our scattered disk-fed control simulation shows a more tightly clustered particle distribution, characterized by $\langle i\rangle \approx 9 \mathrm{deg}$ and $i_{\mathrm{rms}} \approx 19 \mathrm{deg}$. Thus, taken at face value, our calculations suggest that the presence of a significant IOC could partially obfuscate the dynamical signal generated by Planet Nine.

The difference in the statistical measures of orbital clustering between inward-injected IOC objects and outward-scattered Kuiper Belt objects within the context of P9 simulations begs the question of whether or not this discrepancy can be attributed to a difference in particle count among the various numerical experiments. A cursory examination of our simulation suite, however, suggests that this is unlikely to be the case. Out of the 2440 objects initialized within the full set of our IOC-fed simulations, a total of 260 particles attain orbits that satisfy the aforementioned observability criteria at some time in excess of $t>2$ Gyr. The same is true for 227 (out of 1000 initial) particles in our control simulation, implying that the dynamical footprints depicted in the top and bottom panels of
Figure 4 are derived from a comparable number of independent tracers.

In order to quantify the statistical significance of these differences further, we computed the three measures of dynamical confinement- $f_{\varpi},\langle i\rangle,\langle i\rangle / i_{\mathrm{rms}}$-for each IOC-fed model independently, and computed the likelihood that values as low as these can arise within the context for an appropriately resampled control simulation, where the particles are taken to originate within the Kuiper Belt. In particular, we downsampled the control simulation such that the number of independent particles that satisfy our observability criteria at $t>2 \mathrm{Gyr}, N^{\dagger}$, are the same as that in each IOC-fed simulation, and computed the orbital confinement metrics 30 times, randomly reshuffling the particles. We then recorded the fraction of such reshuffled simulations that yield values of $f_{\varpi}$, $\langle i\rangle$, and $\langle i\rangle / i_{\mathrm{rms}}$ equal to or lower than those obtained within the corresponding IOC-fed calculation.

These results are summarized in Table 1 . As can be deduced from these calculations, it is improbable that the differences in the synthetic Kuiper Belts sourced from inward injection versus outward scattering are spurious. In particular, although the inclination-node clustering generated in IOC-fed calculations are in some cases consistent with our control simulation, 
apsidal clustering in these simulations is consistently inferior. To illustrate this point further, we show the $\Delta \varpi$ distributions of simulated particles in the left panel of Figure 5, where the control and IOC-fed simulations are shown with black and multicolored curves, respectively.

A second important result of our numerical experiments is that steady infusion of IOC material into the Kuiper Belt can enhance the prevalence of scattered disk objects with semimajor axes in excess of $a \gtrsim 1000 \mathrm{au}$. That is, although the semimajor axis distribution of dynamical footprints shown in Figure 4 peaks in the $a \sim 250-500$ au range, the tail of the distribution extends beyond $a \gtrsim 3000$ au, as shown in the right panel of Figure 5. Relative to the results of our control simulation, these IOC-fed calculations can readily yield a factor of $\sim 5$ enhancement in the semimajor axis distribution of distant KBOs at $a=2000 \mathrm{au}$ and more than an order of magnitude increase at $a=3000 \mathrm{au}$. Thus, an extended dispersion of KBO semimajor axes may indirectly hint at the operation of P9facilitated modulation of the IOC.

Simultaneously, however, it must be understood that this determination is preliminary. Recall that while the initial conditions of our IOC-fed calculations are generated through self-consistent numerical experiments, the initial conditions of our control simulation were chosen in an ad hoc manner. Because the starting semimajor axis distribution of our control calculation is truncated at $800 \mathrm{au}$, the results may be underestimating the breadth of the true semimajor axis distribution that can be generated through P9-induced evolution of the scattered disk. On the other hand, if the intrinsic surface density of the scattered disk diminishes more steeply than $\Sigma_{\mathrm{SD}} \propto 1 / r$ (as is suggested, for example, by the results of Nesvorný et al. 2017), then our control simulation is likely to be overestimating the extent of the high- $a$ tail of the orbital distribution. A distinct degeneracy relevant to the surface density profile of distant icy bodies arise from the possibility that the presence of the classical Oort cloud may generate an uptick in TNOs with $a \gtrsim 1000$ au that is completely independent of P9-facilitated evolution. Therefore, more precise calculations that account for the existence of the classical Oort cloud and employ a self-consistent estimate of the initial conditions of the scattered disk, are necessary to confidently elucidate the difference between the semimajor axis distributions formed by IOC-fed and scattered disk-sourced populations of distant TNOs.

\section{Conclusion}

In this work, we have carried out a series of numerical experiments in an effort to quantify the injection of inner Oort cloud material into the distant Kuiper Belt via P9-induced dynamical evolution. To this end, we have constructed a model IOC, accounting for the gravitational effects of the Sun's birth cluster (Section 3), and have followed the long-term evolution of this population of distant debris, subject to perturbations from the giant planets, passing stars, galactic tide, and P9 (Section 4). The results of our simulations are readily summarized: if Planet Nine exists, then it facilitates steady variations in the orbits of $a \gtrsim 1000$ au IOC bodies, bringing a fraction of them into the fold of the observable component of the distant scattered disk. Much like the conventional members of the long-period Kuiper Belt, these injected objects exhibit P9-driven orbital confinement. However, the degree of clustering is considerably weaker.
This discrepancy carries important observational consequences. Because the extent of apsidal clustering of distant objects increases with $e_{9}$ (Batygin et al. 2019), contamination of the distant Kuiper Belt by poorly confined IOC objects implies that a more eccentric and distant Planet Nine may be required to explain the data, than is insinuated by the existing literature. However, the magnitude to which a best-fit P9 orbit must be modified depends on a yet-unconstrained fraction of KBOs that are sourced from the IOC. Irrespective of this notion, it is important to note that Planet Nine's eccentricity cannot be enhanced by a large margin without violating other constraints, including the overproduction of highly inclined scattering objects that ensues for sufficiently high values of $e_{9}$, as pointed out by Kaib et al. (2019). Cumulatively, the proofof-principle results reported herein introduce an important, additional degree of uncertainty in our efforts to pin-down the orbit of Planet Nine.

We are thankful to Alessandro Morbidelli, Darryl Seligman, Juliette Becker, Fred Adams, David Nesvorný, and Eduardo Marturet for insightful discussions. We are additionally grateful to David Nesvorný for sharing his implementation of Galactic tide accelerations. We thank the anonymous referee for providing a thorough and insightful report. K.B. is grateful to Caltech, the David and Lucile Packard Foundation, and the Alfred P. Sloan Foundation for their generous support.

\section{ORCID iDs}

Konstantin Batygin (10 https://orcid.org/0000-0002-7094-7908 Michael E. Brown (iD https://orcid.org/0000-0002-8255-0545

\section{References}

Adams, F. C. 2010, ARA\&A, 48, 47

Batygin, K., Adams, F. C., Batygin, Y. K., et al. 2020, AJ, 159, 101

Batygin, K., Adams, F. C., Brown, M. E., et al. 2019, PhR, 805, 1

Batygin, K., \& Brown, M. E. 2016, AJ, 151, 22

Batygin, K., \& Morbidelli, A. 2017, AJ, 154, 229

Bernardinelli, P. H., Bernstein, G. M., Sako, M., et al. 2020, PSJ, 1, 28

Brasser, R., Duncan, M. J., \& Levison, H. F. 2006, Icar, 184, 59

Brasser, R., Duncan, M. J., Levison, H. F., et al. 2012, Icar, 217, 1

Brown, M. E. 2017, AJ, 154, 65

Brown, M. E., \& Batygin, K. 2016, ApJL, 824, L23

Brown, M. E., \& Batygin, K. 2019, AJ, 157, 62

Chambers, J. E. 1999, MNRAS, 304, 793

Clement, M. S., \& Kaib, N. A. 2020, AJ, 159, 285

Hadden, S., Li, G., Payne, M. J., et al. 2018, AJ, 155, 249

Haisch, K. E., Lada, E. A., \& Lada, C. J. 2001, ApJL, 553, L153

Heisler, J., \& Tremaine, S. 1986, Icar, 65, 13

Heisler, J., Tremaine, S., \& Alcock, C. 1987, Icar, 70, 269

Hillenbrand, L. A., \& Hartmann, L. W. 1998, ApJ, 492, 540

Kaib, N. A., Pike, R., Lawler, S., et al. 2019, AJ, 158, 43

Kaib, N. A., Roškar, R., \& Quinn, T. 2011, Icar, 215, 491

Khain, T., Batygin, K., \& Brown, M. E. 2018, AJ, 155, 250

Khain, T., Becker, J. C., \& Adams, F. C. 2020, PASP, 132, 124401

Lada, C. J., \& Lada, E. A. 2003, ARA\&A, 41, 57

Levison, H. F., Morbidelli, A., Van Laerhoven, C., et al. 2008, Icar, 196, 258

Li, G., Hadden, S., Payne, M., et al. 2018, AJ, 156, 263

Madigan, A.-M., Zderic, A., McCourt, M., et al. 2018, AJ, 156, 141

Morbidelli, A., \& Levison, H. F. 2004, AJ, 128, 2564

Morbidelli, A., \& Nesvorný, D. 2020, in The Trans-Neptunian Solar System, ed. D. Prialnik, M. A. Barucci, \& L. Young (Amsterdam: Elsevier), 25

Napier, K. J., Gerdes, D. W., Lin, H. W., et al. 2021, arXiv:2102.05601

Nesvorný, D. 2018, ARA\&A, 56, 137

Nesvorný, D., Vokrouhlický, D., Dones, L., et al. 2017, ApJ, 845, 27

Porras, A., Christopher, M., Allen, L., et al. 2003, AJ, 126, 1916

Saillenfest, M. 2020, CeMDA, 132, 12

Scholtz, J., \& Unwin, J. 2020, PhRvL, 125, 051103

Sefilian, A. A., \& Touma, J. R. 2019, AJ, 157, 59 
Shankman, C., Kavelaars, J. J., Bannister, M. T., et al. 2017, AJ, 154, 50 Sheppard, S. S., \& Trujillo, C. 2016, AJ, 152, 221

Sheppard, S. S., Trujillo, C. A., Tholen, D. J., et al. 2019, AJ, 157, 139
Trujillo, C. A., \& Sheppard, S. S. 2014, Natur, 507, 471

Wiegert, P., \& Tremaine, S. 1999, Icar, 137, 84 